\title{
Musical hallucinations: a case report
}

\author{
Rajiv Weerasundera
}

\section{Summary}

Musical hallucinations as an isolated symptom is an extremely rare presentation. The case of an elderly female presenting with musical hallucinations in the absence of any other demonstrable pathology is reported, discussing the evaluation of possible differential diagnoses and the management of symptoms.

\section{Introduction}

Hallucinations were defined by Esquirol in 1838 as 'a thorough conviction of a perception of a sensation when no external object, suited to excite the sensation, has impressed the senses' (1). They are frequently encountered in the clinical practice of Psychiatry where they occur in all sensory modalities. Among them, auditory hallucinations are common and their diagnostic significance is determined mostly by its content, the presence or absence of organic pathology and the level of insight (2). The relationship between auditory hallucinations and a wide spectrum of acute and chronic organic disorders such as delirium, dementia, cerebral infections, focal neurological lesions and brain neoplasms as well its association with intoxication or withdrawal from substances is well established (3). However, musical hallucinations comprising of instrumental music or songs and occurring in isolation is an extremely rare phenomenon (4). This case report is of an elderly female presenting with musical hallucinations with no other demonstrable pathology related to the symptoms.

\section{Case report}

An eighty four year old widow presented with the 'complaint' of hearing music and the singing of hymns of several weeks duration. She described the experience as pleasant but sought intervention only because the persistence of symptoms was distracting her from engaging in other tasks. The music and the hymns were similar to those sung at her wedding over fifty year earlier.

She had been widowed over twenty years ago and had been living by herself since then. She was the mother of three adult children and enjoyed good relationships with them. She had no past psychiatric history. Her medical co-morbidities included hypertension, hypothyroidism and osteoporosis for which she was being medicated. A few weeks prior to her presentation she suffered an upper respiratory tract infection; her medical history immediately preceding her presentation was otherwise uneventful.
On assessment, she was a pleasant elderly female whose distress due to her symptoms was confined to being distracted from other tasks. Her speech was relevant and coherent and she was euthymic. There were no other perceptual disturbances or other psychotic phenomena. She was well oriented with no evidence of cognitive impairment, scoring 24/30 on Mini Mental State Examination. She was aware that hearing musical sounds was unusual, was puzzled by this and was seeking an explanation, reassurance and intervention for her symptoms. Physical examination, including an ear examination revealed no abnormalities.

\section{Management}

Investigations undertaken included a Full Blood Count, Blood Urea and Electrolytes, Thyroid and Liver functions tests, Syphilis serology, Vitamin B12, Folate and Vitamin D levels, the results of which were unremarkable except for a slightly elevated Thyroid Stimulating Hormone (TSH) level.

An Echocardiogram (ECG) and Electroencephalogram (EEG) revealed no abnormalities. A CT scan of the brain revealed small vascular infarcts in the temporal region which were unlikely to be of recent origin.

Following referrals to a neurologist and an Ear, Nose and Throat (ENT) surgeon, it was established that there was no neurological or ENT pathology that the symptoms could be attributed to. Psychiatric diagnoses such as delirium, dementia and late onset schizophrenia were briefly considered and excluded in the absence of their respective supporting clinical features. A dose increase in her thyroxine restored her euthyroid status but her symptoms persisted.

The patient was commenced on quetiapine $100 \mathrm{mg}$ daily. The initial response was satisfactory with the hallucinations diminishing in frequency and intensity. However, they re-emerged after a few weeks. She was then commenced on risperidone $2 \mathrm{mg}$ daily and has remained symptom free.

\section{Discussion}

Auditory hallucinations characterised by songs, tunes, melodies and rhythms are considered as musical 
hallucinations and historical reports of this phenomenon date back to Baillarger in 1846 (5). It has also been suggested that eighteenth century Austrian composer Joseph Hayden suffered from musical hallucinations (6).

There is no accepted classification of musical hallucinations (4) but the condition has been known by many names: Musical Ear Syndrome, musical hallucinosis, the Oliver Sacks syndrome and the auditory Charles Bonnet syndrome (7). The latter draws an analogy with the Charles Bonnet syndrome, where patients present characteristically with visual hallucinations, preserved awareness of their visual disturbance and an absence of other psychotic symptoms (8).

Musical hallucinations are rare as an isolated symptom in the absence of other pathology, with fewer than 150 reported cases (9).

More commonly musical hallucinations have been reported associated with hearing impairment, epilepsy and psychosis (10). Focal brain lesions and intoxication due to a wide variety of substances have also been implicated (4). In studies of patients presenting with isolated musical hallucinations, the female sex and the elderly appear to be over-represented (11).

Most descriptions of musical hallucinations reveal that the hallucinations have a personal significance and often relate to a previous experience of the patient (12), a feature observed in this patient as well. This has led to the concept that musical hallucinations are the result of a 'parasitic memory', which proposes that they originate from traces of memory retained from previous experiences which cannot be unlearned (10).

It has also been proposed that musical hallucinations are associated more with a right (or non-dominant) hemispheric aetiology (13), this hypothesis being consistent with the right (or non-dominant) hemisphere being more dominant in the perception of music (14). However, the precise pathophysiology of musical hallucinations remains unclear. Brain imaging studies have suggested an associated dysfunction of the temporal cortex (15) but emerging evidence indicates a more heterogeneous causation (4).

As a result of the unclear origin of musical hallucinations, the treatment of this condition remains mostly symptomatic. Given the small numbers of reported cases, systematic studies are yet to be carried out but relief has been obtained with the use of antipsychotics and anti-depressants (4), anti-convulsants (16) and donepezil (17). Individual considerations and the potential side effects of medication are critical factors in the choice of medication.

This patient presented with a rare clinical phenomenon and commoner clinical conditions associated with auditory hallucinations had to be excluded before a definite diagnosis of musical hallucinations could be arrived at. This highlights the significance of a comprehensive assessment, the need to liaise with other specialties when dealing with unusual presentations and the importance of an awareness of the uncommon phenomenology associated with psychiatric disorders.

\section{Declaration of interest}

None declared

Rajiv Weerasundera, Senior Lecturer,

Faculty of Medical Sciences,

University of Sri Jayewardenepura

E-mail: rajivweerasundera@yahoo.com

\section{References}

1. Esquirol JED. Des maladies mentales. Paris: Baillie; 1838.

2. Lowe GR. The phenomenology of hallucinations as an aid to differential diagnosis. Br J Psychiatry 1973; 123:621-33.

3. Fricchione GL, Carbone L, Bennett WI. Psychotic disorder caused by a general medical condition, with delusions: secondary "organic" delusional syndromes. Psychiatr Clin North Am. 1995; 18:363-378.

4. Evers S, Ellger T. The clinical spectrum of musical hallucinations. J Neurol Sci. 2004; 227(1):55-65.

5. Berrios GE. Musical hallucinations. A historical and clinical study. Br J Psychiatry. 1990; 156:188-94.

6. Gordon AG. Seeking Haydn's secrets. Cerebrovasc Dis 1999; 9:54.

7. Blom JD. A dictionary of hallucinations. New York: Springer; 2010

8. Brucki SMD, Takada, LT, Nitrini, R. Charles Bonnet syndrome: case series. Dementia \& Neuropsychologia 2009; 3(1):61-67

9. Blom JD, Sommer IEC. Hallucinations: Research and Practice. New York: Springer; 2012

10. Keshavan MS, Davis AS, Steingard S, Lishman WA. Musical hallucinosis: a review and synthesis. Neuropsychiatry Neuropsychol Behav Neurol 1992;5:211 -23.

11. Pasquini F, Cole MG. Idiopathic musical hallucinations in the elderly. J Geriatr Psychiatry Neurol 1997; 10:11 -4 .

12. Sacks O. The man who mistook his wife for a hat. London: Duckworth; 1985.

13. Berrios GE. Musical hallucinosis: a statistical analysis of 46 cases. Psychopathology 1991;24:356- 60.

14. Bever TG, Chiarello RJ. Cerebral dominance in musicians and non-musicians. Science 1974; 185:5379.

15. Kasai K, Asada T, Yumotot M, Takeya J, Matsuda H. Evidence for functional abnormality in the right auditory cortex during musical hallucinations. Lancet 1999; 354:1703-4.

16. Holroyd S; Sabeen S: Successful treatment of hallucinations associated with sensory impairment using gabapentin. J Neuropsychiatry Clin Neurosci 2008; 20:364-366

17. Ukai S; Yamamoto M; Tanaka M et al.: Donepezil in the treatment of musical hallucinations. Psychiatry Clin Neurosci 2007; 61:190-192 